UCRL-ID-129009

\title{
High Energy-Density Science on the National Ignition Facility
}

\author{
E. M. Campbell \\ R. Cauble \\ B. A. Remington
}

August 1, 1997

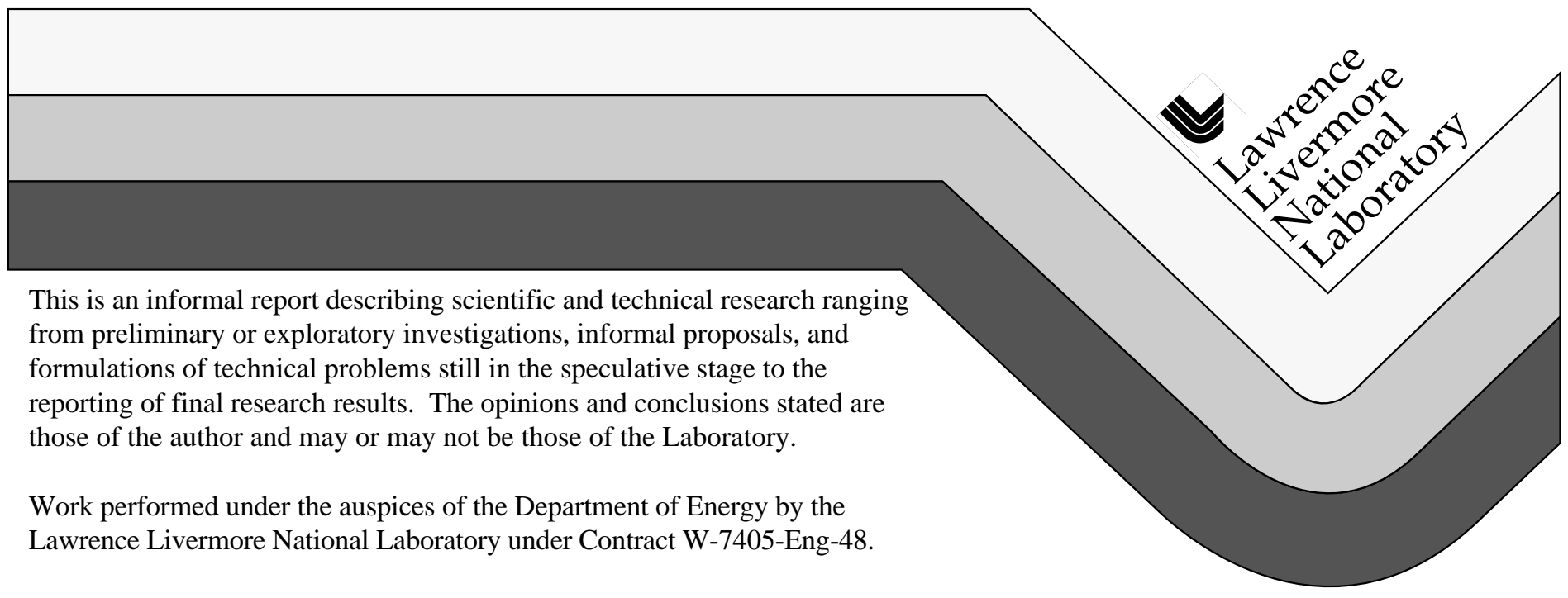




\section{DISCLAIMER}

This document was prepared as an account of work sponsored by an agency of the United States Government. Neither the United States Government nor the University of California nor any of their employees, makes any warranty, express or implied, or assumes any legal liability or responsibility for the accuracy, completeness, or usefulness of any information, apparatus, product, or process disclosed, or represents that its use would not infringe privately owned rights. Reference herein to any specific commercial product, process, or service by trade name, trademark, manufacturer, or otherwise, does not necessarily constitute or imply its endorsement, recommendation, or favoring by the United States Government or the University of California. The views and opinions of authors expressed herein do not necessarily state or reflect those of the United States Government or the University of California, and shall not be used for advertising or product endorsement purposes.

This report has been reproduced directly from the best available copy.

Available to DOE and DOE contractors from the Office of Scientific and Technical Information

P.O. Box 62, Oak Ridge, TN 37831

Prices available from (615) 576-8401, FTS 626-8401

Available to the public from the

National Technical Information Service

U.S. Department of Commerce

5285 Port Royal Rd.,

Springfield, VA 22161 


\title{
HIGH ENERGY-DENSITY SCIENCE ON THE NATIONAL IGNITION FACILITY
}

\author{
E. M. Campbell, R. Cauble, and B. A. Remington \\ Lawrence Livermore National Laboratory, University of California, \\ P. O. Box 808, Livermore, CA 94551
}

\begin{abstract}
The National Ignition Facility, as well as its French counterpart, Le Laser Megajoule, have been designed to confront one of the most difficult and compelling problem in shock physics - the creation of a hot, compressed DT plasma surrounded and confined by cold, nearly degenerate DT fuel. At the same time, these laser facilities will present the shock physics community with unique tools for the study of high energy density matter at states unreachable by any other laboratory technique. Here we describe how these lasers can contribute to investigations of high energy density matter in the areas of material properties and equations of state, extend present laboratory shock techniques such as highspeed jets to new regimes, and allow study of extreme conditions found in astrophysical phenomena.
\end{abstract}

\section{INTRODUCTION}

The goal of inertial confinement fusion (ICF) is to heat a small capsule of DT fuel, compressing it with multiple staged shocks to densities and temperatures capable of sustaining thermonuclear ignition for the purposes of supporting major national activities: maintaining relevant scientific expertise in the absence of nuclear testing and the development of electrical energy production from fusion. (1] The heating will be done with multi-beam, megajouleclass lasers built with advanced but available technology. Lasers will be the driving source for ICF ignition because they can deliver the necessary high power on target in a precisely staged manner, thereby controlling the adiabat of the fuel. Two such lasers, the National Ignition Facility (NIF) in the US[2] and Le Laser Megajoule (3) in France[LM], will come on-line during the first decade of the $21 \mathrm{st}$ century.

A typical ignition target will be a thin spherical lowatomic-number (low-Z) shell surrounding a solid DT shell; DT gas will fill the interior. The conditions necessary for ignition of the target require that the fuel be compressed to very high density $\left(10^{3}-10^{4}\right.$ times liquid) and that a central spark region with a temperature of $10 \mathrm{keV}$ be formed.[4] In order to efficiently compress the fuel, multiple carefully-timed strong shocks (1-100 Mbar) are used so that at ignition the fuel is at enormous pressures but not at high temperature; in fact the fuel will be degenerate; i.e., the fuel temperature will less than the Fermi temperature Thus in order to prepare a capsule for ignition, one needs a cold, dense fuel stell enclosing a $10 \mathrm{keV}$ hot spot at pressures near 100 Gbar (Fig. 1). This is a most interesting, and possibly the most difficult, extant shock physics problem. The lasers that will used to create these conditions will be highly flexible, unique facilities for producing and diagnosing matter in a range of extreme conditions in the laboratory, high energy-density regimes that have never before been experimentally explored.

These lasers will use either direct or indirect drive to compress the ignition capsule. These two methods are now, and will be, used in high energy-density shock experiments. Direct drive involves irradiating a target directly with one or more laser beams. At an intensity $I$ the pressure produced in a low- $Z$ material is given by $P($ Mbar $) \approx$ few times $\left[1\left(10^{14} \mathrm{~W} / \mathrm{cm}^{2}\right) / \lambda(\mu \mathrm{m})\right]^{2 / 3} .[5]$ 


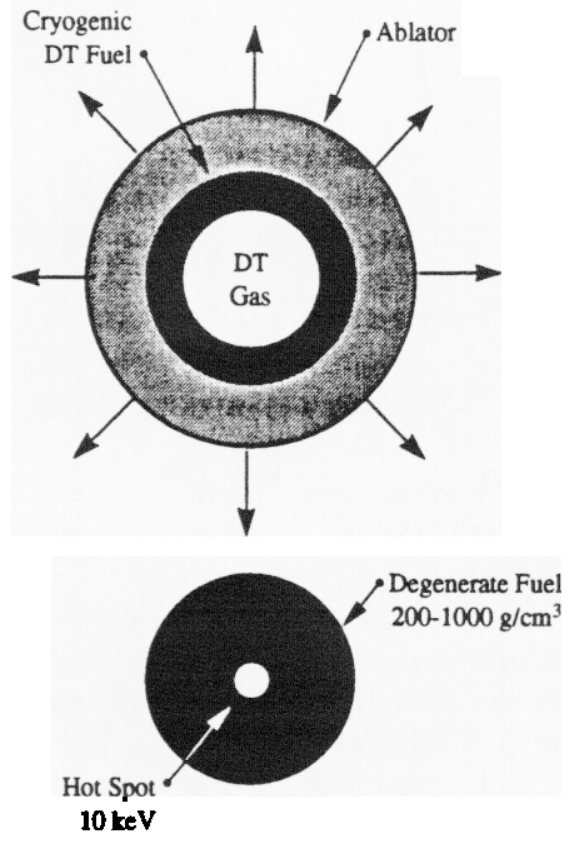

FGURE 1. Schematic compression of an ICF ignition target showing heating of the ablator and corresponding rocket-like implosion of fuel. The resulting ignition configuration is also depicted. Only precisely staged strong shocks from a carefully shaped drive can lead to ignition.

Therefore shocks of 10's of Mbar are possible even with moderate energy lasers, although the necessary intensity is usually achieved over a small spot and for only a very short time duration. Lasers with high energy are needed for most relevant high energy-density experiments. The indirect drive technique uses a high- $Z$ hohiraum into which the laser beams are focused. The resulting $x$-rays, contained in the hohlraum and characterized by a radiation temperature $T_{r}$ are used as the driving source. The pressure generated in the hohiraum is $\approx 10^{4} T_{r}(\mathrm{keV})^{3.5}$ Mbar.[6] With indirect drive, more beams can be used and shock pressures of $\sim 100 \mathrm{Mbar}$ are common on present facilities. NIF hohlraum pressures will be higher, near $500 \mathrm{Mbar}$, and on larger spatial scales. Already indirectly-driven foils ermployed in the same manner as flyer plate shock experiments have unambiguously produced shock pressures of $750 \mathrm{Mbar}$ [7]

Beyond the field of ICF, kilojoule-class lasers like Nova[8] at Lawrence Livermore National Laboratory have been used to investigate fundamental aspects of high energy-density science. The following sections discuss a few areas of high energy-density physics that are being explored using Nova. These range from Mbar-pressure equation of state measurements to studies of hydrodynamic processes in supemovas. In each case the advantages of performing similar experiments on NIF or LMJ are described. An important point to emphasize is that not all high energy-density experiments on NIF will be designed and performed by staff members of the national laboratories. A fraction of the shots will be set aside for use by "outside" users, i.e., university researchers. At the time of this writing a similar plan is being proposed for the LMJ.

\section{MBAR PRESSURE EQUATIONS OF STATE}

The experimental and theoretical investigation of the equations of state (EOS) of materials at high energy density are of interest not only in ICF, but also in astrophysics and other related fields. Strongly shocked matter can be a strongly coupled mixture of molecular, atomic, and ionic species that is extremely difficult to model. Nevertheless EOS tables that include this regime must be constructed not only to design and interpret ICF target data, but also to study the evolution of large planets and stars. This puts a real premium on EOS data in the multi-Mbar regime. Most such data have been generated by nuclear explosions. $[9,10]$

Lasers are capable of producing Mbar shocks but until recently laser-induced shock waves have proved difficult to use for Mbar EOS measurements.[11] Several challenges must be overcome when using lasers in such experiments. In order to reduce uncertainties in the data it is necessary to have a large, spatially uniform, steady-state shock front driven for as long as possible. These are energy considerations, not just intensity considerations. For example, the energy required to maintain a given uncertainty in shock speed at pressure $P$ varies as $P^{5 / 2}$.[12] Preventing preheat of the sample is a stringent requirement since heating of the sample prior to it being shocked changes the initial sample density in an unknown way. Since laser deposition can produce very high temperatures $(\sim \mathrm{keV})$, preheat is a major concern for laserdriven experiments. Finally diagnostics are needed that have few- $\mu \mathrm{m}$ spatial and few-ps temporal resolutions. These are formidable challenges to laser-driven EOS measurements but are not insurmountable.[13] 


\section{Indirectly-Driven Absolute Hugoniots of Polystyrene and Be}

EOS measurements have also be made using indirect drive where higher pressures have been obtained. Because $x$-rays in the hohlraum undergo multiple reflections, the shock generated in a package - a kind of shock tube - attached to the hohlraum is very uniform. There is the additional advantage of having a much lower temperature $x$-ray source, reducing the potential for preheating of a sample. The primary disadvantage of using a hohlraum is that the hohlraum fills with plasma and the laser entrance holes close within a few ns; thus shock measurements must be made within this time duration or corrections made for slowing of the shock in the sample.

The Hugoniots of polystyrene ( $\mathrm{CH}$ ) and beryllium were examined using indirect drive as the source for the shock. Eight beams of the Nova laser were focused into a 3-mm-long by 1.6-mm-diameter gold hohlraum; the EOS package was attached to the side of the hohlraum. [20] See Fig. 3a. Additional beams irradiated a metal foil; $x$-rays from this backlighter were used to radiograph the shock moving through the package. The package consisted of two sections, a pusher of bromine-doped $\mathrm{CH}, \mathrm{CH}(\mathrm{Br})$, close to the hohlraum and the EOS sample attached to the $\mathrm{CH}(\mathrm{Br}) . \mathrm{CH}(\mathrm{Br})$ is opaque to the backlighter $\mathrm{x}$-rays whereas the samples are not. In the same manner as the $\mathrm{D}_{2}$ experiment, $U_{p}$ in the sample was obtained by following the interface motion. The shock front was revealed as the boundary between the highly transmissive unshocked sample and the less transmissive (higher density) shocked sample. The shock speed $U_{s}$ was obtained by following this boundary. Since both $U_{s}$ and $U_{p}$ were measured, the Hugoniot data were absolute data. Data on $\mathrm{CH}$ was obtained from 10 Mbar to $40 \mathrm{Mbar}[14]$, showing that the EOS for $\mathrm{CH}$ from the well-known Sesame EOS library[21] reproduced the data rather well.

Absolute beryllium Hugoniot data were obtained by the same method at 12 and $14 \mathrm{Mbar}$. Fig. 3b shows these data compared with impedance match data from nuclear-explosion-driven experiments $[9,10]$ and Sesame[21]. The absolute laser data lend confidence to the relative underground results.

These results were produced using a kilojoule-class laser. A megajoule-class laser will allow directly driven (a)

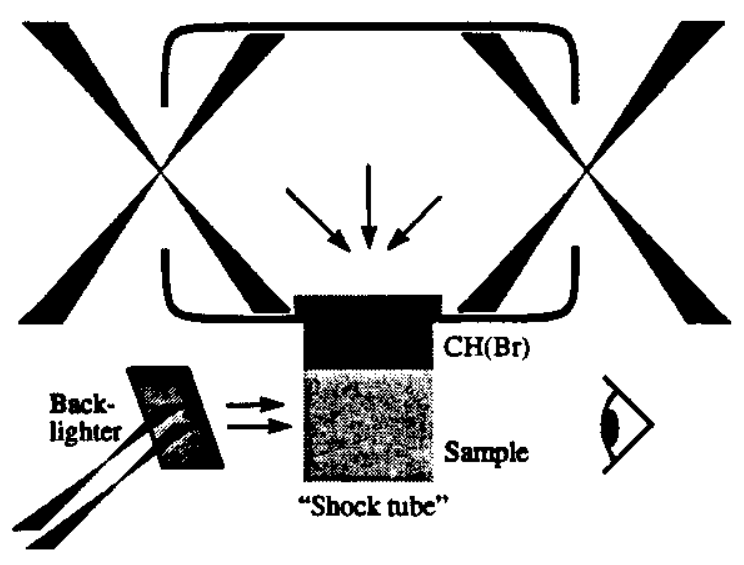

(b)

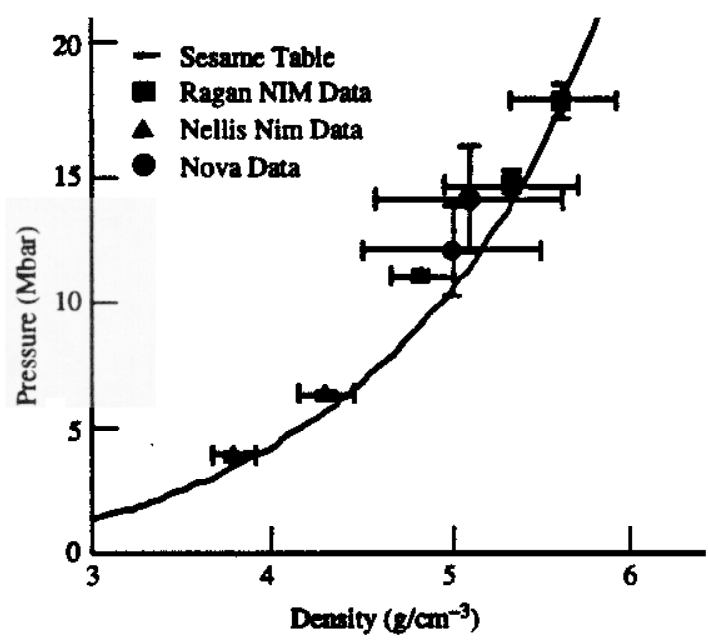

FIGURE 3. a) Experimental setup for the Nova measurement of the Hugoniot of low-Z solids by indirect laser drive employing a gold hohlraum. The experimental package was a two-section shock mbe mounted to the side of the hohlraum. The measurements were made with streak radiography using $x$-rays from a laser-heated backlighter. b) Absolute beryllium Hugoniot data[20] compared with Sesame[21] and nuclear impodance match data $[9,10]$.

shock experiments up to 50 Mbar over longer drive times (up to $20 \mathrm{~ns}$ ) and larger spot sizes. It will be possible to perform absolute EOS measurements with improved accuracy. Scaling of optimized hohlraums from Nova to NIF are expected to allow $1-2 \%$ precision EOS measurements at pressures greater than $1 \mathrm{Gbar}$ in 


\section{Indirectly-Driven Absolute Hugoniots of Polystyrene and Be}

EOS measurements have also be made using indirect drive where higher pressures have been obtained. Because $x$-rays in the hohlraum undergo multiple reflections, the shock generated in a package - a kind of shock tube - attached to the hohlraum is very uniform. There is the additional advantage of having a much lower temperature $x$-ray source, reducing the potential for preheating of a sample. The primary disadvantage of using a hohlraum is that the hohlraum fills with plasma and the laser entrance holes close within a few ns; thus shock measurements must be made within this time duration or corrections made for slowing of the shock in the sample.

The Hugoniots of polystyrene ( $\mathrm{CH}$ ) and beryllium were examined using indirect drive as the source for the shock. Eight beams of the Nova laser were focused into a 3-mm-long by 1.6-mm-diameter gold hohlraum; the EOS package was attached to the side of the hohlraum. [20] See Fig. 3a. Additional beams irradiated a metal foil; $x$-rays from this backlighter were used to radiograph the shock moving through the package. The package consisted of two sections, a pusher of bromine-doped $\mathrm{CH}, \mathrm{CH}(\mathrm{Br})$, close to the hohlraum and the EOS sample attached to the $\mathrm{CH}(\mathrm{Br}) . \mathrm{CH}(\mathrm{Br})$ is opaque to the backlighter $\mathrm{x}$-rays whereas the samples are not. In the same manner as the $\mathrm{D}_{2}$ experiment, $U_{p}$ in the sample was obtained by following the interface motion. The shock front was revealed as the boundary between the highly transmissive unshocked sample and the less transmissive (higher density) shocked sample. The shock speed $U_{s}$ was obtained by following this boundary. Since both $U_{s}$ and $U_{p}$ were measured, the Hugoniot data were absolute data. Data on $\mathrm{CH}$ was obtained from 10 Mbar to $40 \mathrm{Mbar}[14]$, showing that the EOS for $\mathrm{CH}$ from the well-known Sesame EOS library[21] reproduced the data rather well.

Absolute beryllium Hugoniot data were obtained by the same method at 12 and $14 \mathrm{Mbar}$. Fig. 3b shows these data compared with impedance match data from nuclear-explosion-driven experiments $[9,10]$ and Sesame[21]. The absolute laser data lend confidence to the relative underground results.

These results were produced using a kilojoule-class laser. A megajoule-class laser will allow directly driven (a)

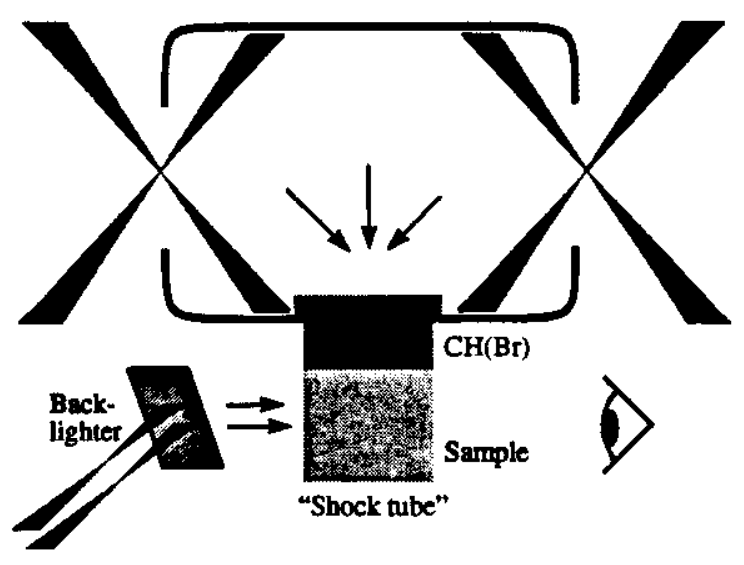

(b)

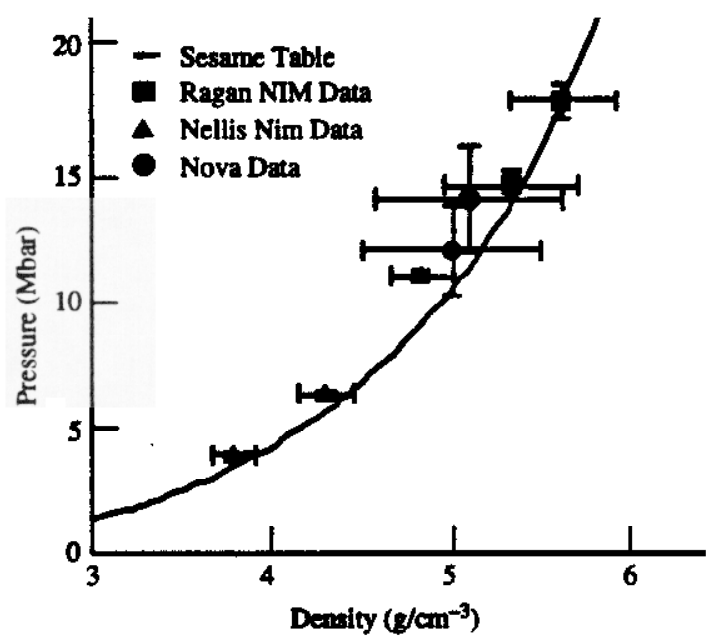

FIGURE 3. a) Experimental setup for the Nova measurement of the Hugoniot of low-Z solids by indirect laser drive employing a gold hohlraum. The experimental package was a two-section shock mbe mounted to the side of the hohlraum. The measurements were made with streak radiography using $x$-rays from a laser-heated backlighter. b) Absolute beryllium Hugoniot data[20] compared with Sesame[21] and nuclear impodance match data $[9,10]$.

shock experiments up to 50 Mbar over longer drive times (up to $20 \mathrm{~ns}$ ) and larger spot sizes. It will be possible to perform absolute EOS measurements with improved accuracy. Scaling of optimized hohlraums from Nova to NIF are expected to allow $1-2 \%$ precision EOS measurements at pressures greater than $1 \mathrm{Gbar}$ in 
an impedance match arrangement. [22] Further development of laser-driven flyer foils should allow few-percent relative EOS measurements near 10 Gbar.

\section{HYDRODYNAMIC MIX AT HIGH ENERGY DENSITY}

Matter motion at high energy density can be susceptible to hydrodynamic instabilities. In an extreme example, Nova has been used to create high-Machnumber jets ( $M=15$ to 30$)$ by using a laser-driven "shaped charge."[23] Instabilities are especially problematic in ICF: mixing of cold fuel into the central hot region can reduce the capsule burn performance or entirely quench the bum.[4] The three most common hydrodynamic instabilities are the acceleration-driven Rayleigh-Taylor (RT) instability, its shock analog the Richtmyer-Meshkov (RM) instability, and the shear-induced Kelvin-Helmboltz (KH) instability. High energy-density hydrodynamic instabilities are not only a factor in ICF, but they are also observable in astrophysical objects (see Section IV). The RT instability can occur at an interface between a low density fluid and a high density fluid where the lighter fluid is accelerated into the heavier. In the linear regime, initial perturbations in the interface will grow exponentially in time with a growth rate $\gamma$ $(\mathrm{Ag} / \lambda)^{1 / 2}$, with $A$ being the density-dependent Atwood number, $g$ the acceleration, and $\lambda$ the perturbation wave length. In the nonlinear asymptotic limit, the interface evolves into bubbles of the lighter fluid rising at their terminal velocity and spikes of the heavier fluid falling through the lighter fluid.

One long-used method to investigate shockinduced mixing is the use of shock tubes.[24] Here, the acceleration is impulsive, the pressures are typically a few bar, the compression is low, and there is no radiation or ionization involved. High explosives generate pressures up to 200-300 kbar[25] and gas guns can generate pressures up to a few Mbar, but with modest compression.[26] Like extraordinary shock tubes, large lasers like Nova and NIF can produce extreme accelerations $\left(10^{13}-10^{14}\right.$ earth gravities) and pressures of 100's of Mbar. Such lasers can achieve high growth factors, large compressions, and high levels of radiation flow and ionization in arbitrary geometry.
There have been a number of experiments to investigate high energy-density hydrodynamic instabilities using a laser drive.[6,27-29] Because of their flexibility, as evidenced in preparing precisely prescribed ICF pulse shapes, lasers can provide the variety of conditions necessary to probe the evolution of RM and RT instabilities. One excellent example is the recent experimental verification by Marinak et al.[30] of the differences between two-dimensional (2D) and three-dimensional (3D) RT-induced perturbation growth. A precisely formed perturbation was pre-imposed on one side of a plastic foil doped with bromine $\mathrm{CH}(\mathrm{Br})$. This foil was placed across an opening on a Nova laser hohlraum which was used to create the $x$-ray drive that ablatively accelerated the foil. The experimental arrangement is similar to that in Fig. 3a, except that the backlighter is positioned above the hohiraum and the detector below so that radiographic images obtained are face-on rather than side-on. Perturbations at the ablation front grow during foil acceleration due to the RT instability. The three perturbations studied all had the same magnitude wavevector, $k=\left(k_{x}{ }^{2}+k_{y}\right)^{1 / 2}$, where $k=2 \pi / \lambda$, and the same amplitude, differing only in their shape: 3D square $k_{x}=k_{y}$,3D stretched $k_{x}=3 k_{y}$, and 2D ripple $k=k_{x}=k_{2 D}$. The images shown in Fig. $4 a$ are timeresolved, face-on, in-flight radiographs taken of the accelerated planar foils. Dark regions correspond to spikes, bright regions to bubbles. The growth versus time of the fundamental mode Fourier amplitudes of the perturbations are shown in Fig. 4b. In the linear regime, all throe modes grow at the same rate, as expected from linear theory, since they all have the same magnitude wavevector. But in the nonlinear regime, the 3D square mode grows the largest, the $2 \mathrm{D}$ ripple grows the least, and the 3D stretched perturbation falls in between. This result can be understood by noting that in the asymptotic limit of terminal bubble velocity, the buoyancy is exactly balanced by the kinematic drag.[31] At the bubble tip, the ratio of drag over buoyancy is smallest for the square mode; this shape has the highest terminal bubble velocity and grows the fastest. The energy of Nova is sufficient to just begin to see these effects. The energy available on NIF will allow 3D experiments to be pushed well into the nonlinear regime. Furthermore we will also be able to perform experiments on NIF with fully multimode 3D foils, not possible on Nova. 

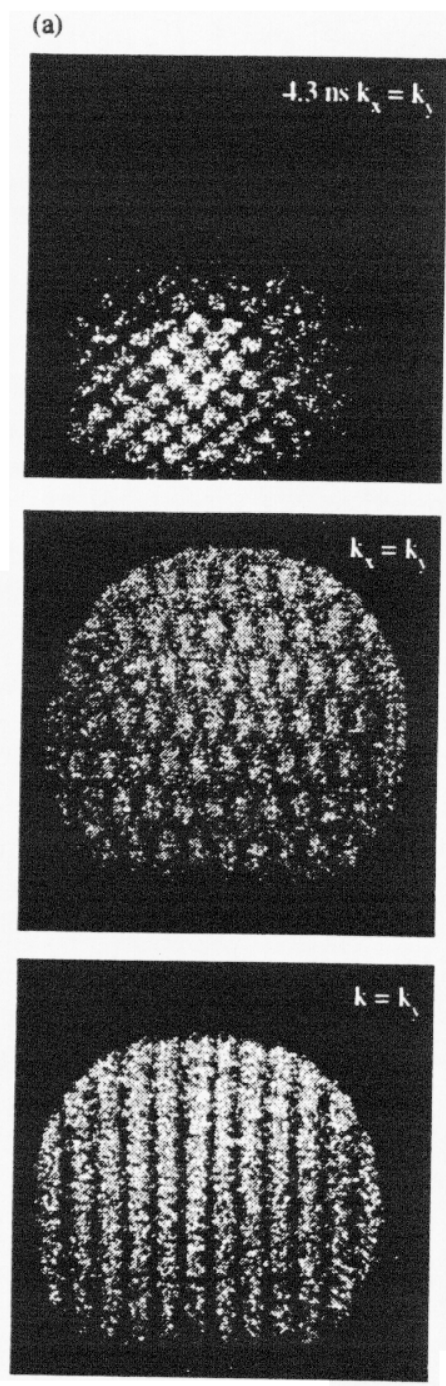

Three-dimensional effects in RT development can also be seen in convergent geometry, the situation encountered in ICF. This is evident in preliminary Nova experiments in which a 3D square $\left(\mathrm{k}_{\mathrm{x}}=\mathrm{k}_{\mathrm{y}}\right)$ mode perturbation was inscribed on the outer surface of a dopedplastic hemisphere and, similar to the planar RT experiments described above, the hemisphere was mounted on the wall of a hohlraum, facing inwards.[32] Ablation of the outer surface by the $x$-ray drive inside the hohlraum causes the hemisphere to radially accelerate. As in the planar case, the perturbations grow as the plastic target is accelerated. But since the hemisphere converges as it is driven, the perturbation wavelength $\lambda$

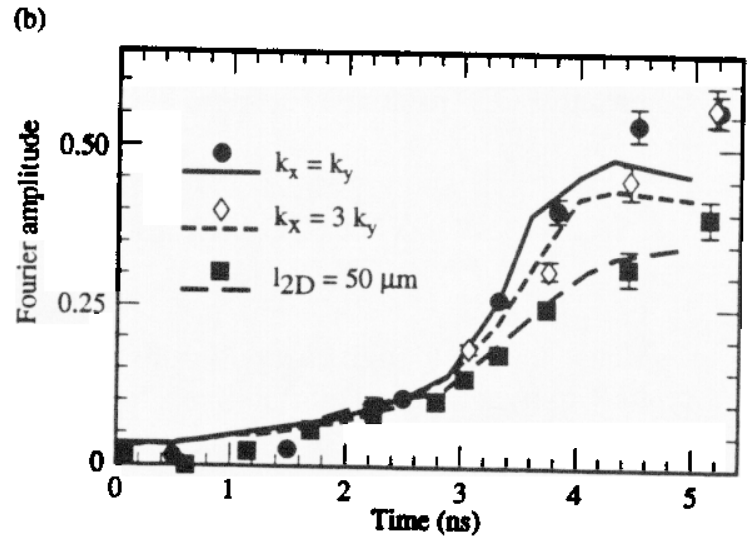

FIGURE 4. a) Face-on radiographs of three different planar Rayleigh-Taylor targets driven indirectly by Nova lasers. The experiment used a hohraum as in Fig. 3a, except that the backlighter was positioned above the hohlraum and the reconting device, an $x$-ray framing camera, was positioned below. The three shapes are 3D square $k_{x}=k_{y}, 3 D$ stretched $k_{x}=3 k_{y}$, and $2 D$ ripple $k=k_{x}=k_{20}$. b) Plot of pertubation Fourier amplitude vs. time for the three shapes showing that the 3D square mode grows fastest

decreases as the shell accelerates. Recalling that the RT growth rate $\sim \lambda^{-1 / 2}$, RT growth was expected to be larg$e r$ in the linear regime in the convergent case and to saturate sooner. This is indeed what was observed. Although the convergence ratio $R_{o} / R$, where $R_{o}$ is the initial hemisphere radius and $R$ the final radius, was only about two, RT growth entered the nonlinear regime. Had the foil been planar, the growth would still be in the linear regime at the times observed. Megajoule-class lasers will be required to drive these 3D experiments well into the nonlinear regime especially, as in the case of planar RT, with multimode targets. The ability to perform fully 3D experiments to study high energy-density hydrodynamics finds application not only in ICF but also in astrophysics, as shown in the following section.

Hydrodynamics at high pressure can also be studied in the solid state. Careful tailoring of the drive pulse in a bohiraum can result in Mbar-pressure shock waves in a metallic targets without melting the target. Recent experiments have shown that $\mathrm{RT}$ growth in solid $\mathrm{Cu}$ is reduced because of strength inherent in the solid.[33] Those experiments also showed that the RT growth reduction could not be explained by extrapolating to Mbar pressures material strength models known to be 
carrect only in the kbar regime. Thus, without these data, Mbar-pressure material strength in $\mathrm{Cu}$ would have been underestimated by a factor of 30 . As a complement to these integral experiments, high pressure shock processes in solids can be investigated in sizu by timeresolved $x$-ray diffraction.[34] As a condensed matter sample deforms under shock loading, the lattice spacing changes and thus the Bragg diffraction angle. Monitoring the diffraction angle as a shock propagates through the sample allows the structure of the shock front to be examined. In siru diffinction of a solid shockcompressed to 300 kbar bas recently been reported.[35] NIF will be able to dive still larger pressures as well as provide additional and higher flux backlighters for more careful measurements.

\section{HIGH ENERGY LASERS AND SUPERNOVAS}

Because of enormous gravitational and nuclear forces, many astrophysical objects are indeed high energy-density phenomena. High energy lasers can contribute to the understanding of astrophysical processes. Exploding stars can create high speed hydrodynamical jets similar to those already described.[36] Supernovas exhibit a range of high energy-density hydrodynamical features that can be scaled to experiments on megajoule-class lasers. Below we describe two experiments developed to investigate supemova phenomena: (1) hydrodynamic-instability-driven core mixing during the SN1987A explosion and (2) strong shock formation in the SN1987A remnant evolution.[37]

\section{SN1987A Mix Experiments}

Gravitational stratification leads to a model of a supernova (SN) progenitor star that is a series of concentric shells with the lightest element, hydrogen, forming the outer envelope, helium just below the $H$ layer, and so on to an iron core. A SN explosion occurs when the Fe core undergoes catastrophic gravitational collapse; a strong radial (rebound) shock is driven outward through the star. At the H/He boundary, the postshock density, temperature, and pressure are about 2.3 $\mathrm{g} / \mathrm{cm}^{3}, 6 \mathrm{keV}$, and $75 \mathrm{Gbar}$. Much effort has been invested in developing models to understand the underlying processes of SNs. Most efforts have focused on one-dimensional (1D) stellar evolution models, treating multidimensional hydrodynamics effects with prescriptions from mixing length theory. Observations of SN1987A highlighted two distinct defects with these models.[38]

A primary means of evaluating models is the SN light curve, the total luminosity of the $\mathrm{SN}$ versus time. The SN luminosity plummets sharply immediately after the explosion as ejected gas hydrodynamically cools due to expansion. Subsequently the luminosity exhibits a broad peak as the heat wave due to radicactive decay of the core diffuses out of the star. In SN1987A, however, the core becarne visible much earlier than 1D diffusion models predicted. [39] The reason was unexpected mixing of the core with the stellar atmosphere sitting on top of the core. Looking at just the $\mathrm{H}$ and $\mathrm{He}$ layers, the boundary between the shells is Richtmyer-Meshkov (RM) unstable as the shock crosses the interface. Further, after shock passage the heavier He decelerates into lighter $\mathrm{H}$ and the interface is RT-unstable so that initial density perturbations continue to grow. The observations from SN1987A provided compelling evidence of the need for two-dimensional (2D) stellar models.[40] Multi-dimensional SN simulations indeed showed that RT-induced mix led to earlier emengence of core radiation compared to $1 \mathrm{D}$ models.[41]

Given the rapid progress in SN modeling over the last ten years, it is reasonable to ask about the accuracy of these multi-dimensional codes. Can they be benchmarked in a meaningful way? For example, 2D modeling predicts peak core velocities of $<2000 \mathrm{~km} / \mathrm{s}$ for SN1987A; however, Doppler broadening shows core velocities that are much higher, $>3000 \mathrm{~km} / \mathrm{s}$. [42] This is an indication that even 2D modeling is insuffcient and 3D codes must be used.

Noting, somewhat remarkably, that we can scale supernova hydrodynamics to the millimeter scales of laser-driven experiments, atempts to simulate SN hydrodynamics have been performed on the Nova laser using a hohlraum $x$-ray drive with $\mathrm{Cu}$ and $\mathrm{CH}_{2}$ as surrogates for $\mathrm{He}$ and $\mathrm{H}$ respectively.[37] The indirectly driven shock tube arrangement (as in Fig. 3a) was utilized. The flexibility inherent in laser energy delivery makes a scaled representation of the correct shockplus-deceleration drive possible. In the experiment, side-on radiographs of the interface show how far and how fast bubbles and spikes form due to RM followed 
by $\mathrm{RT}$ instability. These images are compared to code predictions. Employing a single mode 2D surface (see Fig. 3), gross features of the experiment are reproduced by the simulations.[43] However there is insufficient drive energy available with Nova to allow these experiments to be done in a multimode 3D configuration or experiments in (the correct) exploding geometry. However, NF will have sufficient energy to drive more realistic experiments.

\section{SN1987A Remnant Collision Experiments}

SN1987A is now evolving into the early remnant stage; a 1994 optical image is shown in Fig. 5. The expanding SN ejecta is the central bright spot, surrounded by an assembly of nebular rings, the origin of which is a mystery. Gaseous SN ejecta, moving at $-10^{3}-10^{4} \mathrm{~km} / \mathrm{s}$, will collide with the inner circumstellar ring in 5-10 years in a celestial display that may shed light on the nature of the rings.[40] Using this collision to probe the nature of the rings depends

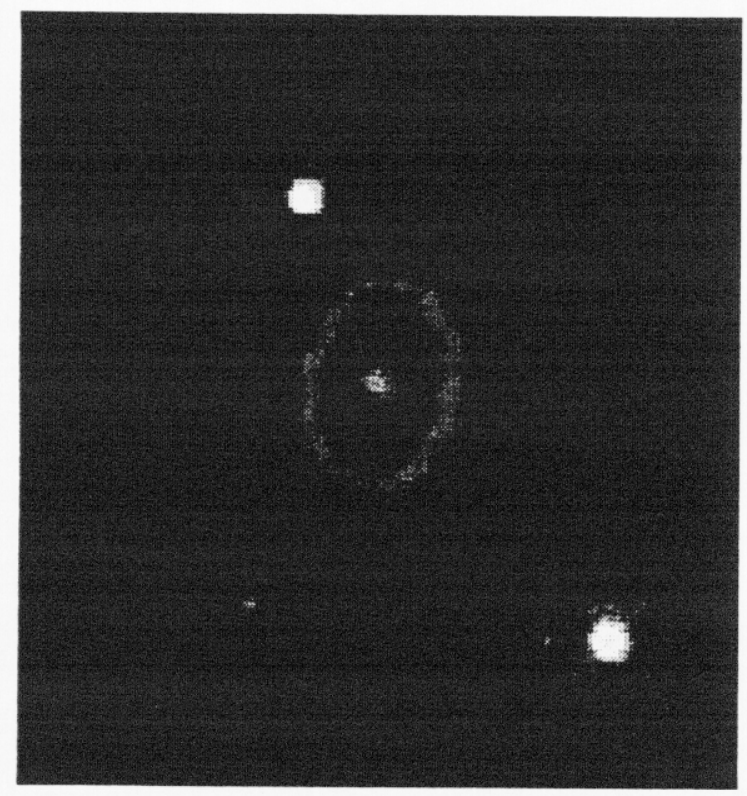

FIGURE 5. An image of SN1987A obtained by the Hubble Telescope in Feb. 1994. The expanding supemova ejecta is the central dot. The rings are planetary nebulae of uncertain origin on correctly interpreting observations of a complex, energetic, hydrodynamically unstable collision between two interstellar plasmas. Simulations are being performed now to predict the outcome of the collision.

When the two plasmas collide, shocks will be launched forward into the ring and backward into the SN ejecta. Cooling of the strongly shocked plasma by radiation causes the compressed ejecta to collapse to an even higher density leading to strong RT growth at the contact discontinuity. [44] Qualitatively different mixing evolves depending on the density profile of the ejecta and the initial evolution of the contact discontinuity. It is therefore beneficial to test various models experimentally prior to the collision in order to assess how astrophysics codes handle the complex radiative plasma dynamics.

An experiment has been designed as a proxy to the stellar collision. [37] In the experiment, $\mathrm{CH}(\mathrm{Br})$ is used to provide a surrogate for the $\mathrm{SN}$ ejecta; $\mathrm{SiO}_{2}$ aerogel foam represents ambient plasma and a solid $\mathrm{CH}$ foil corresponds to the ring plasma. Attached to a Nova hohlraum, $x$-rays drive a 50 Mbar shock into the $\mathrm{CH}(\mathrm{Br})$, ejecting plasma into a gap between the $\mathrm{CH}(\mathrm{Br})$ and the foam. The ejecta stagnates in the foam driving shocks forward into the foam and backward into the ejecta. Side-on $x$-ray backlighting reveals the positions of the shocks and the relative shock densities. Experiments on Nova have successfully produced and imaged both shocks. However, the shocks are not so strong that cooling radiation is a factor in the Nova experiment. Further, longer $x$ ray drives are needed to evince the expected RT development in the plasma. NIF will provide the drive energy to overcome both of these difficulties.

\section{SUMMARY}

A primary mission of megajoule-class lasers such as the NIF and LMJ will be to compress, inertially confine, and initiate nuclear fusion in DT capsules. This approach to ICF ignition requires a flexible, highenergy facility capable of producing conditions of matter unattainable by other laboratory methods while simultaneously allowing experimental investigation of shocked, high energy-density matter. We have described several examples of high energy-density 
experimental investigations that have already been initiated on present-day kilojoule-class lasers and how those studies will be extended on NIF and LMJ:[45] We examined EOS measurements from 1 to 100's of Mbar, production of high-Mach-number jets, and the growth and development of hydrodynamic instabilities in planar and convergent geometries that reveal the limitations of two-dimensional models. Further we described how high energy lasers can be used to investigate shocks in astrophysical phenomena, in particular supemovas. Perhaps most important, experiments at NIF will be performed not only by the staff at US national laboratories, but also in collaboration with and by members of the academic shock physics community, serving to invigorate the field of high energydensity physics.[46]

\section{ACKNOWLEDGMENTS}

This work was performed under the auspices of the U.S. Department of Energy by the Lawrence Livermore National Laboratory under contract No. W-7405-ENG-48

\section{References}

1.E M. Compbell, N. C. Holmes, S. B. Lbby, R A. Reaington, and E. Tellex, b epper in Leer Part. Benms (Deceuber, 1997 ).

2. J. A. Plener, EM Compbell, and WJ. Hogen, Fusion Techoology 26, 755 (1994).

3. M Andre, "Conceptual Design of the Fiench LMJ L sere," Firex SPIE intemionel Conference on Solid Strte Lmens for Appliavion to ICF, Montercy, (1995).

4. Jobn Lindl, Ptys. Piames 2, 3933 (1995).

5. Th Lower and R. Sivel, Contrib. Placme Phys. 33, 355 (1993).

6. B. A. Remingwon at al., Ftys. Pisumes 2, 241 (1995).

7.R Cuble et al, Phys. Rev. Lett. 70, 2102 (1993).

8 EM. Compbell, Leser Pant Besms 9, 209 (1991).

9. C. E Regen II, Phys. Rev. A 29, 1391 (1984).

10. W. J. Nellis, J. A. Marixity, A. C. Mitchell, and N. C. Hotmes, J. Apph Physics 82, 2225 (1997).

11. M Evass a al., Lasers Part Beans 14, 113 (1996).

12. N. C. Halmes, pivete communication.

13. Y. M. Gupta and S. M. Sherme, Science 277, 909 (1997).

14. R. Couble et al., Phys. Plasmes 4, 1857 (1997).

15. W. J. Nellis a al, J. Chem. Phys. 79, 1480 (1983).

16. Y. B. Zel'dovich and Y. P. Raizer, Physics of Shock Waves and High-Temperanure Hydrodynamic Phenomena (Acodemic Press, New York, 1966).
17. L. B. Da Silva et al., Phys. Rev. Lex. 78, 483 (1997).

18.N. C. Holmes, M. Ross, and W. J. Nellis, Phys. Rex B 52, 15835 (1995).

19. K. S. Budil et al., "Cherecterization of Laser-Driven Shock Waves using Interferometry." to appear in Inertial Confinement Fusion Qwarterly Reporn, LLNL repart (1997).

20. R. Cruble at al, "Absouhre Equation of Stute Dath in the 10-40 Mber (1-4 Tpa) Regime," submined o Phys. Rev. Leth (1997).

21. S. P. Lyon and J. D. Johnson, "SESAME: The Los Alamos National Laboratory Equation of State Database," Los Alemos National Laboretory report LA-UR-92-3407 (1992).

22. N. Lenden, private communication.

23. P. L. Miller a al., "Shock-hydrodynamic Experiments on Nova," Procectings of the 20th Intemetional Symposium on Shock Waves," Pasedena (1995).

24. M. Vever and B. Sturtevene, Shock Whes 4, 247 (1995); $R$. Bonszea and B. Sturtevent, Fhys. Fluids 8, 2496 (1996).

25. R.F. Benjamin and J.N. Fritz, Phys. Flids 30, 331 (1987).

26. For example, N.C. Holmes et al, Appl. Phys. Leth 45, 626(1984).

27. K. Budil at al., Phys. Rev. Leth 76, 4536 (1990); G. Dimonte ex al, Phys. Rev. Lett. 74, 4855 (1995); T.A Peyser et al, Phys. Rev. Lett. 75, 2332 (1995).

28. W. Hising et al., Phys. Rev. Lett 78, 3876 1997); Phys. Piosmas 4, 1832 (1997).

29. K Nithihara end H. Saknama Phys. Rev. Let. 65, 432 (1990).

30. M. Marinak et al., Phys. Rev. Let. 75, 3677 (1995).

31. J. Hecht et al., Imer and Purt. Beams 13, 423 (1995).

32. S.G. Glendinning et al., in Proceedings of the 6eh linemational Workshop on the Physics of Compressible Turbulent Mixing, Mrseille, in press (1997).

33. D.H Kelmen, in the proceedings of the Geh Intemational Wortatop on the Physics of Compressible Turbulent Mixing, Manceille (1997).

34. J. S. Wodk et al., J. Appl. Prys. 68, 4531 (1990).

35. D.H. Kolantri, et al, "Truedene X-ray Difiraction Uned to Diegnowe Figh Preasure Shocted Solids," Anmual Meeting of the Amarican Cyrtellogrephyic Asocintion, St Louis (1997).

36. S. Fe thoove et al, Atron. J. 112, 1141 (1990).

37. B. A. Remington \& el., Phys. Plomes 4, 1994 (1997).

38. Sec, es., L. Hachiou, T. Mussuda, K. Nomoto, T. Shigeyama, Astron Astrophys. Suppl 104, 341 (1994); M. Henext and SE. Wookey, Atrophys. J. 02, 814 (1994).

39 T. Srigeyman and K. Nomon, Astrophys. J. 350, 242 (1990).

40. R. MoCray, Amn Rev. Avron. Astrophys. 31, 175 (1993).

41. D. Ameth, Astrophys. J. 27,932 (1994).

42. B. Fryxell, W. Mnlex, and D. Amet, Astrophys. J. 367, 619 (1991).

43. J. Kene at al., Astrophys. J. 478, L75 (1997).

44. R.A. Chevalier and J.M. Blondin, Astrophys. J. 44, 312 (1995).

45. R. W. Lee, "Science on High Energy Laeen from Today to the

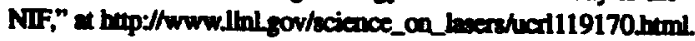

46. See the repart Facility Uac Plan for the Netional Ignition Facility," eds. A. Haver, R Kunfimen, A. Strsengi, T. Hoill, R. Couble, and T. Saito, avilable from US Deperment of Energy, Office of the NIF, 1000 independence Avenie, S.W. Washington, DC 20585. 


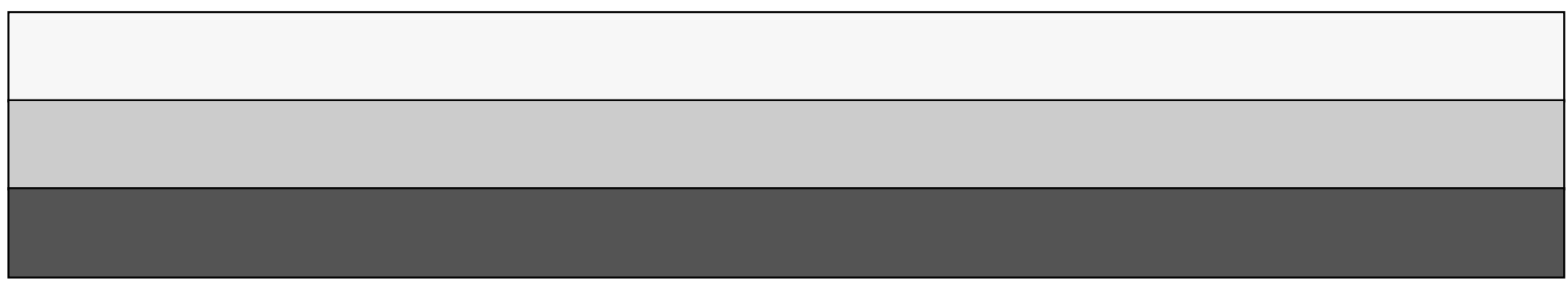

